The Astronomical Journal, 115:2356-2358, 1998 June

(C) 1998. The American Astronomical Society. All rights reserved. Printed in U.S.A.

\title{
AN OLD CLUSTER IN NGC $6822^{1}$
}

\author{
Judith G. COHEN AND JohN P. BlaKeslee \\ Palomar Observatory, Mail Stop 105-24, California Institute of Technology, Pasadena, CA 91125 \\ Received 1998 February 25
}

\begin{abstract}
We present spectroscopy of two clusters in the dwarf irregular galaxy NGC 6822. From these we deduce an age for cluster VII of $11_{-3}^{+4} \mathrm{Gyr}$ and $[\mathrm{Fe} / \mathrm{H}]=-1.95 \pm 0.15$ dex. Cluster VII appears to be an analog of the metal-poor Galactic globular clusters. Cluster VI is found to be much younger and more metal-rich, with an age of approximately $2 \mathrm{Gyr}$. Its derived metallicity, $[\mathrm{Fe} / \mathrm{H}] \approx-1.0 \mathrm{dex}$, is comparable to that of the gas seen today in NGC 6822. The existence of a metal-poor old cluster in NGC 6822 rules out models for the chemical evolution of this galaxy with significant prompt initial enhancement. We find that a star formation rate that is constant with time and is within a factor of 2 of the present star formation rate can reproduce the two points on the age-metallicity relationship for NGC 6822 over the past $10 \mathrm{Gyr}$ defined by these two clusters.
\end{abstract}

Key words: galaxies: abundances — galaxies: dwarf — galaxies: individual (NGC 6822) galaxies: star clusters

\section{INTRODUCTION}

NGC 6822 is a dwarf irregular galaxy in the Local Group. It has several bright clusters found by Hubble (1925), with many more cluster candidates subsequently cataloged by Hodge (1977). While most of them are bright blue (presumably young) clusters, one of them, cluster VII, is sufficiently red that it may be a true globular cluster. The goal of this paper is to establish the age and metallicity of this object.

The star formation history of this galaxy was studied initially by Hodge (1980). Marconi et al. (1995), Gallart, Aparicio, \& Vílchez (1996c), and Gallart et al. (1996a, 1996b) present more modern analyses based on CCD imaging and photometry. Like many dwarf irregular galaxies (Hunter 1982), star formation within NGC 6822 appears to be distributed over isolated spatial regions at any given time, and to vary with time at each position. Hunter (1997) reviews star formation processes in these small galaxies where spiral density waves are absent.

Such studies can only examine in detail the star formation history over the past $3 \mathrm{Gyr}$. Older stars are fainter and are easily swamped by the brighter, younger ones. The existence of a genuinely old globular cluster in such a galaxy would be irrefutable evidence for star formation more than $10 \mathrm{Gyr}$ ago, an issue relevant to the concept of bursting dwarfs introduced by Broadhurst, Ellis, \& Shanks (1988) and Babul \& Rees (1992), among others, to try to explain the origin of the excess of blue galaxies found in deep galaxy counts.

We have recently completed an analysis of the ages and abundances of a large sample of globular clusters in M87 (Cohen, Blakeslee, \& Ryzhov 1998), and we apply these techniques to two of the bright clusters in NGC 6822 to demonstrate that cluster VII is indeed a genuine old globular cluster.

\footnotetext{
${ }^{1}$ Based in large part on observations obtained at the W. M. Keck Observatory, which is operated jointly by the California Institute of Technology and the University of California.
}

\section{SPECTROSCOPY OF TWO CLUSTERS IN NGC 6822}

We obtained spectra with the Low Resolution Imaging Spectrograph (LRIS; Oke et al. 1995) on the Keck II Telescope in 1997 September. We used the same instrumental configuration for NGC 6822 as in our observations of the M87 globular clusters, with a 1".5-wide long slit replacing the multislit masks. By rotating the instrument's position angle, we placed both cluster VI and cluster VII of NGC 6822 in the slit. Three exposures, each $300 \mathrm{~s}$ long, were taken, followed by a flat field.

The line indices for cluster VI and cluster VII in NGC 6822 on the Lick system were measured in a manner identical to that described in our work on M87, and are listed in Table 1. Although $\mathrm{H} \beta$ appears in absorption, the integrated light of cluster VI shows strong emission in $\mathrm{H} \alpha$, indicating immediately that this is a young cluster. The measurements listed in Table 1 do not include any correction for underlying emission in the Balmer lines.

The model grid of Worthey (1994) was used to interpret these measurements in exactly the same way as we did for the M87 globular clusters. We find that cluster VII has $[\mathrm{Fe} / \mathrm{H}]=-1.95 \pm 0.15$ dex on the scale of Zinn (1985) and an age of $11_{-3}^{+4}$ Gyr. This age is consistent with the mean ages of the Galactic and M87 globular clusters, which we found to be in the range 12-15 Gyr.

For cluster VI, we had to extrapolate the models in order to constrain its age and metallicity. Doing so, we find $[\mathrm{Fe} / \mathrm{H}] \approx-1.0 \mathrm{dex}$, age $\approx 2 \mathrm{Gyr}$, but these values are highly uncertain because of the large extrapolation, as the Worthey models only extend down to $8 \mathrm{Gyr}$ at this low metallicity and to $[\mathrm{Fe} / \mathrm{H}]$ of -0.22 at this young age. However, the models clearly indicate an age less than $5 \mathrm{Gyr}$, and we expect that our value of 2 Gyr may be an overestimate, as a consequence of our ignoring any emission in the $\mathrm{H} \beta$ line.

The metallicity we have derived for cluster VII is consistent with that expected from the mean relation between globular cluster metallicity and host galaxy luminosity (van den Bergh 1975; Harris 1991; Perelmuter 1995). Although the precise form of this relationship is still in dispute, for 
OLD CLUSTER IN NGC 6822

TABLE 1

INDICES ON THE LICK SYSTEM FOR Two CLUSTERS IN NGC 6822

\begin{tabular}{lccccccccr}
\hline \hline Cluster & $\begin{array}{c}\mathrm{H} \beta \\
(\AA)\end{array}$ & $\begin{array}{c}\mathrm{Mg}_{1} \\
(\mathrm{mag})\end{array}$ & $\begin{array}{c}\mathrm{Mg}_{2} \\
(\mathrm{mag})\end{array}$ & $\begin{array}{c}\mathrm{Mg} b \\
(\AA)\end{array}$ & $\begin{array}{c}\mathrm{Fe} 5270 \\
(\AA)\end{array}$ & $\begin{array}{c}\mathrm{Fe} 5335 \\
(\AA)\end{array}$ & $\begin{array}{c}\mathrm{Na} \mathrm{D} \\
(\AA)\end{array}$ & $\begin{array}{c}\mathrm{TiO}_{1} \\
(\mathrm{mag})\end{array}$ & $\begin{array}{r}\mathrm{H} \alpha \\
(\AA)\end{array}$ \\
\hline VI $\ldots \ldots . .$. & 3.75 & 0.017 & -0.002 & 0.308 & 0.682 & 0.610 & 1.02 & 0.004 & -6.45 \\
VII $\ldots . .$. & 2.70 & 0.032 & 0.004 & 0.581 & 0.731 & 0.810 & 1.12 & 0.005 & 2.27 \\
\hline
\end{tabular}

dwarf galaxies with luminosities similar to that of NGC 6822 , the expected mean metallicity of any old globular cluster system is $\langle[\mathrm{Fe} / \mathrm{H}]\rangle \approx-1.7 \pm 0.2$ dex, with a dispersion of $\sim 0.3$ dex for individual globular clusters. Thus, it appears that cluster VI is a compact, young open cluster, while cluster VII is a typical old globular cluster (more evidence from photometry is given below).

These results support the findings of Gallart et al. (1996a), who concluded that NGC 6822 most likely began forming stars $\sim 12$ Gyr ago from low-metallicity gas and has continued forming them at a slow rate ever since. In particular, our observations immediately eliminate the class of "prompt chemical enrichment" models, considered but deemed unlikely by these authors, wherein a short-lived starburst of massive stars promptly enriches the gas from primordial to a tenth of solar at very early times. Gallart et al. (1996a, p. 1962) state that the discovery of "indicators of the presence of an old, low-metallicity population, such as a horizontal branch for example, would definitely resolve the question." Our observation of an old globular cluster in this galaxy now resolves the issue.

\section{INFERRED METAL-ENRICHMENT RATE}

The mean metallicity of the gas in NGC 6822 today is known from studies of its $\mathrm{H}$ II regions (Lequeux et al. 1979; Pagel, Edmunds, \& Smith 1980; Skillman, Terlevich, \& Melnick 1989). These three papers obtained consistent results of $\mathrm{O} / \mathrm{H} \sim 0.15$ times the solar value. In old metalpoor stars in our own Galaxy, $\mathrm{O} / \mathrm{H}$ is enhanced over $\mathrm{Fe} / \mathrm{H}$ by a factor of $\sim 2$ (Wheeler, Sneden, \& Truran 1989). If we assume this is also the case in NGC 6822, then the current $[\mathrm{Fe} / \mathrm{H}]$ of its gas is approximately $-1.1 \mathrm{dex}$, consistent with the very young age and similar metallicity for cluster VI, while cluster VII has a metallicity some $\sim 7$ times lower. This difference represents the accumulated metal formation by generations of stars in NGC 6822 .

Hodge (1993) reports a current star formation rate (SFR) of $0.021 M_{\odot} \mathrm{yr}^{-1}$ for NGC 6822 based on its global $\mathrm{H} \alpha$ luminosity. From an estimate of the total stellar mass of this galaxy, he concludes that the time-averaged SFR is very close to the current one; in particular, if NGC 6822 has been forming stars for $12 \mathrm{Gyr}$, the inferred mean SFR is $\sim 0.028 M_{\odot} \mathrm{yr}^{-1}$. Gallart et al. (1996b) find an SFR of 0.04 $M_{\odot} \mathrm{yr}^{-1}$ over the past $\sim 200 \mathrm{Myr}$, which they interpret as a stochastic enhancement of roughly a factor of 2 over the mean SFR. They point to Hodge's current value as perhaps indicating that the SFR has once again decreased. In any case, the data indicate that NGC 6822 has been steadily forming stars at a very modest rate of $0.02-0.04 M_{\odot} \mathrm{yr}^{-1}$ throughout its lifetime.

We can ask whether the metal-enrichment rate inferred from our data is consistent with a constant star formation rate approximately equal to the observed one. A naive "closed box" model of chemical enrichment (see, e.g.,
Binney \& Tremaine 1987) predicts that the metallicity of the interstellar gas will increase with time as

$$
Z(t)=Z_{0}-p \ln \left[1-\frac{M_{s}(t)}{M_{s+g}}\right],
$$

where $Z_{0}$ is the initial metal fraction, $M_{s}(t)$ is the mass in stars at time $t, M_{s+g}$ is the constant total mass in stars and gas, and $p$ is the "yield parameter," the fraction of input mass that gets returned to the interstellar gas in the form of heavy elements. The mean SFR is $\left\langle\dot{M}_{s}\right\rangle \equiv M_{s}(t) / \Delta t$. The value of $p$ is fairly uncertain, but a reasonable estimate is $p=0.005 \pm 0.003$ (see, e.g., Rana 1991; Edmunds \& Pagel 1984). Adopting values of $\Delta Z \approx 0.0015 \pm 0.0007$ and $\Delta t \approx 10 \pm 3 \mathrm{Gyr}$ for the difference in metallicity and age between clusters VI and VII, and taking $M_{s+g}$ from Gallart et al., we find a mean SFR in NGC 6822 of $\left\langle\dot{M}_{s}\right\rangle=$ $0.04_{-0.03}^{+0.04} M_{\odot} \mathrm{yr}^{-1}$, consistent with other estimates and with the currently observed SFR. Of course, if infall of primordial gas was important, then in order to produce the same amount of metal enrichment the true time-averaged SFR would have to be higher than our naive estimate.

\section{PHOTOMETRY AND RADIAL VELOCITY OF CLUSTER VII}

The distance modulus for NGC 6822 comes from its Cepheids, originally studied by Kayser (1967), with a recent reanalysis by Visvanathan (1989). We adopt the reddening and distance modulus for this galaxy of Gallart et al. (1996c). The observed colors for cluster VII are $U-B=0.30$ and $B-V=0.85$, with $V=15.78$, while those of cluster VI are $\sim 0.5$ mag bluer in both $B-V$ and $U-B$ (van den Bergh \& Humphreys 1979; Wilson 1992), as expected. The luminosity of cluster VII is then $M_{V}=-8.45$ $\operatorname{mag}\left(L=1.9 \times 10^{5} L_{\odot}\right)$. This corresponds to a luminosity somewhat brighter than that of the peak of the Galactic globular cluster luminosity function, at $M_{V}=-7.35 \mathrm{mag}$ (Zinn 1985).

The unreddened colors for cluster VII are $(B-V)_{0}=0.61$ and $(U-B)_{0}=0.11$. These agree to within the uncertainties of measurement with the colors of metal-poor Galactic globular clusters such as M92, M13, and M3 as tabulated by Reed, Hesser, \& Shawl (1988). Wilson (1992) arrived at the opposite conclusion and asserted that cluster VII is similar to the intermediate-age clusters of the Magellanic Clouds, but she was using a reddening value substantially larger than the one adopted here.

The distribution of the $\mathrm{H}$ I gas in the central region of NGC 6822 has been mapped by Gottesman \& Weliachew (1977), who see a rotating disk, a pattern typical of such galaxies. The observed heliocentric radial velocity from our LRIS spectra of cluster VII is $-52 \mathrm{~km} \mathrm{~s}^{-1}$, which corresponds closely to the $v_{r}$ expected from the $\mathrm{H} \mathrm{I}$, so there is no evidence for a "halo" on kinematic grounds. 


\section{SUMMARY}

We have established from analysis of its spectral features that cluster VII in NGC 6822 appears to be a metal-poor old globular cluster, with an age of $11_{-3}^{+4} \mathrm{Gyr}$ and $[\mathrm{Fe} / \mathrm{H}]=-1.95 \pm 0.15$ dex. Cluster VI in NGC 6822 is found to be much younger and more metal-rich, with an age of approximately 2 Gyr. The metallicity derived for cluster VI, $[\mathrm{Fe} / \mathrm{H}] \approx-1.0 \mathrm{dex}$, is comparable to that of the gas in NGC 6822 at the present epoch. The colors of these two objects are consistent with this interpretation. This eliminates models of the chemical evolution of NGC 6822 that invoke strong prompt initial enrichment and has implications for the idea that bursting dwarf galaxies are the origin of the excess numbers of faint blue objects found in deep galaxy counts. For instance, these results do not support the suggestion (Broadhurst et al. 1988) that occasional strong bursts of star formation in fairly isolated, gas-rich dwarfs are responsible for the excess faint counts, although late- forming dwarf elliptical and spheroidal galaxies that deplete their gas supply in a strong initial burst (Babul \& Rees 1992) are still a possibility. A star formation rate that is constant in time and within a factor of 2 of the present value can reproduce the two known points provided by clusters VI and VII on the age-metallicity relationship for NGC 6822 .

The entire Keck/LRIS user community owes a huge debt to Jerry Nelson, Gerry Smith, Bev Oke, and many other people who have worked to make the Keck Telescope and LRIS a reality. We are grateful to the W. M. Keck Foundation, and particularly its late president, Howard Keck, for the vision to fund the construction of the W. M. Keck Observatory. J. G. C. is grateful for support from NSF grant AST 96-16729. J. P. B. is grateful to the Sherman Fairchild Foundation for support.
Babul, A., \& Rees, M. J. 1992, MNRAS, 255, 346

Binney, J., \& Tremaine, S. 1987, Galactic Dynamics (Princeton: Princeton Univ. Press)

Broadhurst, T. J., Ellis, R. S., \& Shanks, T. 1988, MNRAS, 235, 827

Cohen, J. G., Blakeslee, J. P., \& Ryzhov, A. 1998, ApJ, 496, 808

Edmunds, M. G., \& Pagel, B. E. J. 1984, MNRAS, 211, 507

Gallart, C., Aparicio, A., Bertelli, G., \& Chiosi, C. 1996a, AJ, 112, 1950 1996b, AJ, 112, 2596

Gallart, C., Aparicio, A., \& Vílchez, J. M. 1996c, AJ, 112, 1928

Gottesman, S. T., \& Weliachew, L. 1977, A\&A, 61, 523

Harris, W. E. 1991, ARA\&A, 29, 543

Hodge, P. W. 1977, ApJS, 33, 69 1980, ApJ, 241, 125

ESO), 501 1993, in Dwarf Galaxies, ed. G. Meylan \& P. Prugniel (Garching:

Hubble, E. P. 1925, ApJ, 62, 409

Hunter, D. A. 1982, ApJ, 260, 81 1997, PASP, 109, 937

\section{REFERENCES}

Kayser, S. E. 1967, AJ, 72, 134

Lequeux, J., Peimbert, M., Rayo, J. F., Serrano, A., \& Torres-Peimbert, S. 1979, A\&A, 80, 155

Marconi, G., Tosi, M., Greggio, L., \& Focardi, P. 1995, AJ, 109, 173

Oke, J. B., et al. 1995, PASP, 107, 307

Pagel, B. E. J., Edmunds, M. G., \& Smith, G. 1980, MNRAS, 193, 219

Perelmuter, J. M. 1995, ApJ, 454, 762

Rana, N. C. 1991, ARA\&A, 29, 129

Reed, B. C., Hesser, J. E., \& Shawl, S. J. 1988, PASP, 100, 545

Skillman, E. D., Terlevich, R., \& Melnick, J. 1989, MNRAS, 240, 563

van den Bergh, S. 1975, ARA\&A, 13, 217

van den Bergh, S., \& Humphreys, R. M. 1979, AJ, 84, 604

Visvanathan, N. 1989, ApJ, 346, 629

Wheeler, J. C., Sneden, C. \& Truran, J. W. 1989, ARA\&A, 27, 279

Wilson, C. D. 1992, AJ, 104, 1374

Worthey, G. 1994, ApJS, 95, 107

Zinn, R. 1985, ApJ, 293, 424 